\title{
Michael Wendl
}

\section{Die »angebotspolitische« Wende in der Tarifpolitik Kritik der aktuellen Lohnpolitik der Gewerkschaften ${ }^{1}$}

In den letzten vier Jahren ist die Tarifpolitik der deutschen Gewerkschaften in doppelter Weise unter Druck geraten. Zum einem sind auf der gesamtwirtschaftlichen Ebene, also in makroökonomischer Perspektive, die $\mathrm{Zu}$ wachsraten des Nominallohns insgesamt so niedrig gewesen, daß sie in der mehrjährigen Frist unter dem Anstieg der Arbeitsproduktivität blieben. Zum anderen verstärkt sich der Erosionsprozeß der Flächentarifverträge, so $\mathrm{daß}$ aus Angst vor ihrer Auflösung Lohnkostensenkungen auf der Ebene von Unternehmen und Betrieben ermöglicht werden. Auch auf der mikroökonomischen Ebene sinken somit die Löhne, sowohl durch offene Tarifflucht als auch durch das aus Angst vor dem Ende der Flächentarifverträge akzeptierte »Öffnen« der Tarifverträge für betriebliche Regelungen. Auf dieser Ebene vollzieht sich auch die Angleichung der Effektivlöhne an die Tariflöhne und damit das Schwinden der sog. Lohndrift. In den neuen Bundesländern kann flächendeckend sogar von einem deutlichen Unterschreiten der tatsächlichen Entgelte gegenüber dem tariflichen Niveau gesprochen werden.

Die innergewerkschaftliche Verarbeitung dieser tarifpolitischen Umbrüche geschieht in zwei kontroversen Debatten. Die erste dreht sich um die von Klaus Zwickel initiierte Idee eines »Bündnis für Arbeit«, die zweite um die Frage der Öffnung der Flächentarifverträge für spezielle betriebliche Anwendungen. Hinter beiden Prozessen steht die elementare Frage, ob unter dem Druck der Globalisierung und des verschärften Standortwettbewerbs demokratische Verfahren zur Festsetzung der nationalen Arbeitskosten aufrechterhalten werden können.

1 Der Autor dieses Beitrags hat an den genannten innergewerkschaftlichen Debatten teilgenommen und die Mehrheitsposition entschieden kritisiert (vgl. Wendl 1996a, 1996b). In einer Zeitschrift für kritische Sozialwissenschaft sollten dieser Mangel an Distanz einerseits und die gleichzeitige Kenntnis interner Diskussionen andererseits offen benannt werden. 


\section{Die Kontroverse um das $\gg$ Bündnis für Arbeit«}

Zwickels Vorschlag, im Rahmen eines Bündnisses zwischen Arbeitgebern, Bundesregierung und IG Metall auf mögliche Lohnerhöhungen oberhalb der Preissteigerungsrate $\mathrm{zu}$ verzichten und »befristete Einarbeitungsabschläge« für Langzeitarbeitslose zu akzeptieren, wurde innerhalb wie auBerhalb der Gewerkschaften als entscheidende tarifpolitische Weichenstellung wahrgenommen. Dabei bestand der Richtungswechsel in der Tarifpolitik nicht in dem Gedanken eines tripartistischen Bündnisses zwischen Staat, Unternehmen und Gewerkschaften. Bereits 1993 hatten die DGBGewerkschaften einen von der Bundesregierung vorgeschlagenen Solidarpakt ohne größere interne Kontroversen akzeptiert. Trotz gelegentlich radikaler Rhetorik waren die deutschen Gewerkschaften in ihrer großen Mehrheit seit den 50er fast durchgängig an klassenübergreifender Kooperation interessiert. Die neue Botschaft aus der IG Metall lautete: Wir akzeptieren wenn auch probehalber - die Sichtweise der neoklassischen Lohn- und Beschäftigungstheorie, daß mit (relativen) Lohnsenkungen ein Anstieg des Beschäftigungsgrades verbunden ist. Diese Aussage war auch deshalb überraschend, weil Zwickel in Passagen der gleichen Rede vor der Präsentation der Bündnis-Offerte exakt gegenteilig argumentiert hatte. Gestützt auf Schlußfolgerungen von Untersuchungen des Deutschen Instituts für Wirtschaftsforschung (DIW 1995), teilweise in wörtlicher Übernahme der Formulierungen aus dem Wochenbericht $38 / 95$ des DIW hatte er den $\mathrm{Zu}$ sammenhang von Lohnverzicht und daraus resultierender Aufwertung der DM dargestellt und weiterem Lohnverzicht eine Absage erteilt. Die von Zwickel akzeptierte Übernahme zentraler Aussagen der Standortstudie des DIW macht nur Sinn, wenn das »Bündnis für Arbeit« zunächst als taktisches Manöver verstanden wird. Wird die wirtschaftspolitische Bewertung des DIW inhaltlich akzeptiert, so wird damit eine Politik der relativen Lohnsenkung auf nationaler Ebene ausgeschlossen, da die gewonnenen Wettbewerbsvorteile durch die dadurch provozierte Aufwertung der DM wieder verloren gingen. Die Übernahme der Argumente des DIW zur Standortposition hat daher nur den Charkter einer rhetorischen Pflichtübung. Die Richtigkeit der eigenen Position wird beschworen, um sofort davon abzuweichen und entgegen der politischen Überzeugung zu handeln. Damit wurde die Opferbereitschaft signalisiert, die im Gegenzug von der anderen Seite ebenfalls erwartet wurde. Und genau das hat die andere Seite nicht getan. Sie hat zwar ungeschickt taktiert, aber die eigenen Interessen nicht aufgegeben, sondern gegenüber der Bundesregierung durchgesetzt. Warum unmittelbar danach, von der zustimmend zitierten Sichtweise des DIW abgewichen und die Bereitschaft zum Lohnverzicht signalisiert wird, wird nur aus nachgereichten Begründungen ersichtlich. Wenn die nominale 
Lohnerhöhung deutlich unter dem Anstieg der Arbeitsproduktivität bleibt, soll der Rest der »Produktivitätskomponente《 für die Ausweitung von Arbeits- und Ausbildungsplätzen verwendet werden (Lang/Kuhlmann 1996, 194). Dieses Angebot der Lohnzurückhaltung in Verbindung mit einer Ausweitung des Beschäftigungsgrades kann in einer makroökonomischen Sichtweise nicht überzeugen. Lohnabschlüsse unter dem zu erwartenden Produktivitätsanstieg führen im Rahmen einer kapitalistischen Geldwirtschaft nur dann zu steigender Beschäftigung, wenn sich Fiskal- und Geldpolitik gleichzeitig auf einem expansiven Kurs bewegen. Nur dann wird die Einsparung an Arbeitskosten in zusätzliche Beschäftigung umgesetzt. Darüber vermittelt wird ein niedrigerer Anstieg der Arbeitsproduktivität und damit eine geringere Verdrängung der Lohnarbeit durch Kapital theoretisch möglich. Bezogen auf die Entwicklung der von der Lohnbewegung abhängigen Sozialeinkommen führt das Prinzip der Nettolohnorientierung zu realen Kürzungen bei Renten und Arbeitslosenunterstützungen und verringert insgesamt die konsumtive Nachfrage. Unter den Rahmenbedingungen einer deflationär orientierten Fiskalpolitik und auf ein hohes Realzinsniveau geradezu rücksichtslos fixierten Bundesbankpolitik ${ }^{2}$ konnten die mit der Lohnzurückhaltung verbundenen Beschäftigungshoffnungen aber nicht aufgehen.

In der politischen Reaktion auf die Bündnis-Offerte der Gewerkschaften hat dann der überwiegende Teil der Unternehmensverbände deutlich gemacht, daß die Unterstellung, die Unternehmen selbst hätten ein wirkliches Interesse an einer spürbaren Verringerung der Massenarbeitslosigkeit, naiv und zugleich riskant war, da das Angebot der Gewerkschaften als Zeichen politischer Schwäche, die es auszunutzen gelte, wahrgenommen wurde. Faktisch haben sich die Unternehmensverbände mit dem neuen Entgeltfortzahlungsgesetz im Rücken auch durchgesetzt. Zum einen hat die IG Metall mit einer Nominallohnerhöhung von 1,5\% den bloßen Ausgleich der Preissteigerungsrate für 1997 ohne jegliches Entgegenkommen der Arbeitgeberseite in Sachen Arbeitsplätze akzeptiert, zum zweiten hat sie Kostenentlastungen auch bei der Lohnfortzahlung selbst (Wegfall der Entgelte und Zuschläge für Mehrarbeit) ermöglicht, die den gesamten Arbeitskostenanstieg unter der zu erwartenden Inflationsrate halten. Sie hat daher, folgen wir der sog. Meinhold-Formel (Altvater/Mahnkopf 1993, 68) in der produktivitätsorientierten Lohnpolitik, etwas mehr als den zukünftigen Produktivitätsanstieg für 1997 der Unternehmensseite zugestanden. Damit geht sie sogar deutlich über die lohnpolitische Empfehlung der neoklassischen

2 Die Koordination von Lohn-, Fiskal und Geldpolitik erfordert die Einbindung der Zentralbank in ein $\gg$ Bündnis für Arbeit«. Angesichts der politischen und makroökonomischen Dominanz der Bundesbank disqualifiziert sich ein ohne Einbeziehung dieser Institution vorgeschlagenes Bündnis für mehr Beschäftigung als fachlich inkompetent, egal ob aus neoklassischer, keynesianischer oder marxistischer Sicht. 
Doktrin hinaus. Danach bestimmt sich der beschäftigungsneutrale Lohnerhöhungsspielraum aus dem Anstieg der Arbeitsproduktivität und dem Anstieg der Absatzpreise der Unternehmen (Siebert 1994, 73). Liegt der Anstieg des Nominallohns unter dem Anstieg der Summe dieser beiden Faktoren, kommt es aus neoklassischer Sicht zu einem durch niedrige Löhne bedingten Anstieg der Beschäftigung.

Diese Sichtweise erfährt ihre Faszination dadurch, daß sie die in der Grenznutzentheorie angelegte simple handlungstheoretische Konstruktion, daß mit sinkenden Löhnen die Beschäftigungsneigung des Unternehmers steige, einfach auf die Gesamtwirtschaft überträgt. Dagegen hatten die Gewerkschaften, argumentativ unterstützt durch die traditionelle Unterkonsumtionstheorie der 20er Jahre, bisher eingewandt, daß gegenüber den Produktionskosten relativ sinkende Löhne makroökonomisch gesehen die konsumtive Nachfrage verringern und dadurch zu zusätzlicher unfreiwilliger (»keynesianischer«) Arbeitslosigkeit führen würden. Beide Sichtweisen, also die Löhne als Kostenfaktor oder als Nachfragefaktor im Sinne eines Wirkungszusammenhangs mit dem Beschäftigungsgrad verbinden zu wollen, demonstriert eine eher vorwissenschaftliche Besessenheit, weil die Lohnhöhe für die Frage des Umfangs der Arbeitslosigkeit eine neben anderen Faktoren wie z. B. des Konjunkturzyklus, der Investitionsquote, der Fiskalpolitik, der Geld- und Währungspolitik eher untergeordnete oder auch davon abhängige Rolle spielt. Marx hat auf die der Lohnbewegung eigentümliche Verkehrung hingewiesen:

»Es sind diese absoluten Bewegungen in der Akkumulation des Kapitals, welche sich als relative Bewegungen in der Masse der exploitablen Arbeitskraft widerspiegeln und daher der eigenen Bewegung der letztren geschuldet scheinen. Um mathematischen Ausdruck anzuwenden: die Größe der Akkumulation ist die unabhängige Variable, die Lohngröße die abhängige, nicht umgekehrt.« (MEW 23, 648)

Die Relativierung der beschäftigungspolitischen Funktion der Lohnhöhe erfolgt, um die weitverbreitete Ineinssetzung von mikroökonomisch fundierten Handlungen von Individuen einerseits und darauf basierenden makroökonomischen Konstruktionen der Neoklassik andererseits zu kritisieren. In einer durch Deflationspolitik gekennzeichneten makroökonomischen Konstellation führen ein zu starkes Nachhinken der Lohnentwicklung gegenüber der Produktivität bzw. eine fallende Lohnquote zu steigender Arbeitslosigkeit. Dieser Zusammenhang kann in einer kapitalistischen Geldwirtschaft aber nicht dazu gewendet werden, daß mit relativ steigenden Löhnen die Arbeitslosigkeit entsprechend abgebaut werden kann. Das bedeutet, daß eine expansive Lohnpolitik - wenn sie überhaupt durchzusetzen wäre - keinen Ausweg aus Wirtschaftskrise und Arbeitslosigkeit weist. Das durch Tarifverträge regulierte Arbeitskostenniveau darf nicht so hoch sein, daß die Profitrate im industriellen Sektor zu nahe an den Kapital- 
marktzins gerät und damit die Realkapitalakkumulation einschränkt und es darf nicht so stark sinken, daß die konjunkturelle Abwärtsbewegung durch eine sinkende konsumtive Nachfrage verstärkt wird. In der relativen Starrheit der Tarifverträge nach unten liegt eine wichtige konjunkturstabilisierende Funktion in einer ansonsten labilen Geldwirtschaft. Diese Starrheit der Löhne nach unten wird aber gerade von der Neoklassik kritisiert.

Die Gewerkschaften verhindern durch die nach unten starren Löhne, daß Arbeitsmärkte bei Unternachfrage nach Arbeitskräften wie Gütermärkte mit einem entsprechenden Preisverfall reagieren. Diese Funktionsweise des Arbeitsmarktes sichert nicht nur einen stabilen ökonomischen Anker, sondem wirkt neben dem System der sozialen Sicherung als - wenn auch brüchiger - Garant für ein halbwegs menschenwürdiges Verhalten bei der Suche nach Arbeit. Die offene Konkurrenz unter den Arbeitssuchenden wird erheblich eingeschränkt. Wird diese Regulierung nicht mehr durch ein halbwegs flächendeckendes System von Tarifverträgen gesichert, so müssen - mindestens in demokratischen Gesellschaften - Mindestarbeits- und entlohnungsregeln gesetzlich normiert werden. Die davon Betroffenen werden anders als bei Tarifverträgen an der Erarbeitung und Festlegung dieser Normen aber nicht beteiligt. Es ist gerade das demokratische Verfahren der Tarifpolitik, das ihre Ergebnisse erklärt.

In der »Bündnis für Arbeit«-Debatte haben die Gewerkschaften unter dem Druck erodierender Flächentarifverträge selbst diese Ineinssetzung oder besser Konfusion von Mikro- und Makroökonomie versucht und haben dabei eine - gemessen an der verteilungspolitischen Bilanz - eindeutige tarifund beschäftigungspolitische Niederlage hinnehmen müssen. In der politischen Öffentlichkeit wird die Eindeutigkeit dieser Bilanz allerdings nicht hinreichend wahrgenommen. Der Konflikt im Unternehmerlager um die ta-

3 Den Tarifforderungen und -abschlüsse gehen mehr oder weniger intensive Diskussionen in demokratisch gewählten Tarifkommissionen, bei den jährlichen Lohnrunden auch in Mitgliederversammlungen voraus. Dazu müssen zwei Einschränkungen gemacht werden. Einmal erklärt der demokratische Charakter dieser Verfahren das Defizit einer gewerkschaftlichen Theorie der Lohnpolitik. Hinter den Forderungen steht kein ausgearbeitetes verteilungspolitisches Konzept, sondern demokratisch artikulierte Lebens- und Anerkennungsbedürfnisse. Andererseits versucht der kleine Kreis der Verhandlungsführungen regelmäßig Ansprüche und Erwartungen nach unten zu schrauben, um die Enttäuschungen über das zu niedrige Ergebnis gering zu halten. Grundsätzlich gilt für die beteiligten Gewerkschaftsmitglieder Keynes' Aussage »Die Arbeiter sind daher glücklicherweise, obschon unbewußt, instinktiv vernünftigere Wirtschaftler als die klassische Schule, indem sie sich gegen eine Kürzung der Geldlöhne wehren, selbst wenn der bestehende Realgegenwert dieser Löhne den Grenzanteil der bestehenden Beschäftigung übersteigt, während sie sich einer Kürzung der Reallöhne nicht widersetzen, die mit einer Zunahme der gesamten Beschäftigung verbunden ist« (Keynes 1973, 12). Der daraus resultierenden „Kaufkrafttheorie« des Lohnes wurde aber ebenso unvermittelt eine rationalisierungsfördernde »Kostentheorie « entgegengestellt, wenn es darum ging, politisch nicht opportune, hohe oder egalitäre Lohnforderungen abzuwehren. 
rifpolitisch gescheiterte Durchsetzung des neuen Entgeltfortzahlungsgesetzes einerseits und die Niederlage der Bundesregierung mit diesem nur sehr eingeschränkt geltenden Gesetz verstellen dazu den Blick. Die weitgehende Verhinderung der Umsetzung des Entgeltfortzahlungsgesetzes durch die Kompensation mit niedrigen Nominallohnsteigerungen zählt bei der politischen Bewertung stärker als die rein verteilungspolitische Bilanz, deren Resultate die Beschäftigungskrise verstärken werden.

Eine Formulierung der Zusammenhänge zwischen Lohnhöhe und Arbeitslosigkeit wie sie z. B. durch die modifizierte Philipps-Kurve vorgenommen wird, führt zu irreführenden Konsequenzen, weil die Korrelationen zwischen Nominallohnsteigerung und Arbeitslosigkeit (ursprüngliche Philipps-Kurve) bzw. zwischen Inflation und Arbeitslosigkeit (modifizierte Philipps-Kurve), die unbestreitbar sind, solange es Nominallohnsteigerungen und Arbeitslosigkeit gibt, in der Regel als Kausalzusammenhänge aufgefaßt werden, die sie jedoch nicht sind. ${ }^{4}$ In der Regel werden die lohnpolitischen Forderungen der Gewerkschaften aus mehreren Faktoren gebildet. Grundlage ist die Orientierung am Ausgleich der Preissteigerungsrate, wobei hier nicht der Deflator des Bruttoinlandsprodukts, sondern die Entwicklung der Verbraucherpreise zum Maßstab gemacht und überwiegend nicht vom Netto-, sondern vom Bruttolohn ausgegangen wird. Zusätzlich wird eine Beteiligung am Wirtschaftswachstum oder die Zuwachsrate der Arbeitsproduktivität und (gelegentlich) eine Umverteilungskomponente gefordert bzw. ein Nachholbedarf aus der unzureichenden Steigerung der vorhergegangenen Tarifrunde geltend gemacht. Die Darstellung der Entwicklung der Bruttoarbeitseinkommen seit 1970 zeigt, daß der von der neoklassischen Theorie definierte Lohnerhöhungsspielraum in einer Reihe von Jahren überschritten wurde und zwar überwiegend in Jahren mit steigender Beschäftigung, nur 1974 (einmal in 11 Jahren) fielen sinkende Beschäftigung und Überschreiten des Verteilungsspielraums zusammen. Von der Neoklassik wird ein zeitlich so enger Zusammenhang nicht behauptet, sondern ein Zusammenhang von bis zu drei Jahren angenommen (Siebert 1994, 99). Damit wird dann aber lediglich der konjunkturelle Verlauf auf dem Arbeitsmarkt nachgezeichnet. Vor der Rezession können wegen der gestiegenen Nachfrage nach Arbeitskräften höhere Nominallohnsteigerungen durchgesetzt werden, in der Rezession schwächt sich die Verhandlungsmacht der Gewerkschaften durch die Zunahme der Arbeitslosigkeit. Die durch die Konjunkturentwicklung geprägte $\mathrm{Zu}$ - oder Abnahme der Nachfrage auf dem Arbeitsmarkt bestimmen die

4 Mit Sicherheit gibt es auch eine Korrelation zwischen dem Absatz von Sejdenkrawatten und den Indices der wichtigsten Börsenkurse. Solche Korrelationen bestehen zwischen allen denkbaren jährlich erhobenen ökonomischen Indices. Die mathematisch korrekte Feststellung quantitativer Korrelationen völlig disparater Größen erlaubt aber keine inhaltliche Aussage darüber, warum diese Korrelationen existieren. 
Nominallohnbewegungen im wesentlichen. Dabei haben die Gewerkschaften in den 80ger Jahren und in der ersten Hälfte der 90er den Verteilungsspielraum der produktivitätsorientierten Lohnpolitik bei weitem nicht ausgeschöpft. Sie haben das, was aktuell von ihnen gefordert wird - längerfristige Lohnabschlüsse unter dem Produktivitätsanstieg - bereits mehrfach eingelöst. Insofern wird die skeptische Beurteilung von Glastetter $u$. a., daß in den 80er Jahren Spielräume für mehr Wachstum und Beschäftigung verschenkt wurden, weil die Lohnkostenentlastung zur Nichtweitergabe von Produktivitätszuwächsen in die Löhne und damit zum Ausfall von Kaufkraft für eine hinreichende Konsumnachfrage führte (Glastetter, Högemann, Marquardt 1991, 114ff) im nachhinein plausibel.

\section{Löhne, Preise und Beschäftigung 1970 - 95}

\begin{tabular}{|l|l|l|l|l|l|l|l|l|}
\hline Jahr & $\begin{array}{l}\text { Preis- } \\
\text { steige- } \\
\text { rungs- } \\
\text { raten } \\
\text { BSP }\end{array}$ & $\begin{array}{l}\text { Pro } \\
\text { duk } \\
\text { tivi- } \\
\text { tät }\end{array}$ & $\begin{array}{l}\text { Ver- } \\
\text { lei- } \\
\text { lungs- } \\
\text { spiel- } \\
\text { raum }\end{array}$ & $\begin{array}{l}\text { Brutto- } \\
\text { lohn- } \\
\text { steige- } \\
\text { rung } \\
\text { pro } \\
\text { Besch. }\end{array}$ & $\begin{array}{l}\text { Uber-bzw. } \\
\text { Unterschrei- } \\
\text { tung des } \\
\text { Verteilungs- } \\
\text { spielraums }\end{array}$ & $\begin{array}{l}\text { Lohn- } \\
\text { quote } \\
\text { struk- } \\
\text { turbe- } \\
\text { reinigt }\end{array}$ & $\begin{array}{l}\text { Besch. } \\
\text { Arbeit- } \\
\text { nehmer } \\
\text { i. Tsd. }\end{array}$ & $\begin{array}{l}\text { Verän- } \\
\text { derung } \\
\text { gegen } \\
\text { Vorjahr } \\
\text { in \% }\end{array}$ \\
\hline 1970 & 7,6 & 3,8 & 11,4 & 15,0 & $+3,6$ & 68,0 & 22246 & $+2,2$ \\
\hline 1971 & 8,0 & 2,5 & 10,5 & 11,3 & $+0,8$ & 68,9 & 22605 & $+1,6$ \\
\hline 1972 & 5,3 & 3,8 & 9,1 & 8,2 & $+0,9$ & 69,0 & 22841 & $+1,0$ \\
\hline 1973 & 6,4 & 3,6 & 10,5 & 10,7 & $+0,2$ & 69,7 & 23222 & $+1,6$ \\
\hline 1974 & 7,1 & 1,5 & 8,6 & 10,5 & $+1,9$ & 71,8 & 23036 & $-0,8$ \\
\hline 1975 & 6,0 & 1,1 & 7,1 & 6,2 & $-0,9$ & 71,9 & 22467 & $-2,5$ \\
\hline 1976 & 3,6 & 6,0 & 9,6 & 6,9 & $-2,7$ & 70,2 & 22512 & $+0,2$ \\
\hline 1977 & 3,7 & 2,8 & 6,5 & 6,8 & $+0,3$ & 70,5 & 22686 & $+0,8$ \\
\hline 1978 & 4,3 & 2,0 & 6,3 & 5,3 & $-1,0$ & 69,5 & 22961 & $+1,2$ \\
\hline 1979 & 4,0 & 2,4 & 6,4 & 5,7 & $-0,7$ & 69,4 & 23472 & $+2,2$ \\
\hline 1980 & 4,8 & $-0,2$ & 4,6 & 6,6 & $+2,0$ & 71,6 & 23897 & $+1,8$ \\
\hline 1981 & 4,0 & 0,3 & 4,3 & 4,8 & $+0,5$ & 72,4 & 23907 & $+0,04$ \\
\hline 1982 & 4,4 & 0,6 & 5,0 & 3,9 & $-1,1$ & 72,5 & 23639 & $-1,1$ \\
\hline 1983 & 3,3 & 3,0 & 6,3 & 3,2 & $-3,1$ & 70,4 & 23293 & $-1,5$ \\
\hline 1984 & 2,0 & 2,7 & 4,7 & 3,0 & $-1,7$ & 69,2 & 23351 & $+0,25$ \\
\hline 1985 & 2,2 & 1,2 & 3,6 & 2,9 & $-0,7$ & 68,7 & 23559 & $+0,9$ \\
\hline 1986 & 3,1 & 0,9 & 4,0 & 3,6 & $-0,4$ & 68,8 & 23910 & $+1,5$ \\
\hline 1987 & 2,0 & 1,0 & 3,0 & 3,1 & $+0,1$ & 68,1 & 24141 & $+1,0$ \\
\hline 1988 & 1,6 & 2,9 & 4,5 & 2,9 & $-1,6$ & 67,0 & 24365 & $+0,9$ \\
\hline 1989 & 2,4 & 2,1 & 4,5 & 3,0 & $-1,5$ & 65,8 & 24750 & $+1,6$ \\
\hline 1990 & 3,2 & 2,7 & 5,9 & 4,7 & $-1,2$ & 64,9 & 25460 & $+2,8$ \\
\hline 1991 & 3,8 & 1,9 & 5,7 & 5,9 & $+0,2$ & 64,9 & 25920 & $+1,8$ \\
\hline 1992 & 4,4 & 0,5 & 4,9 & 5,8 & $+0,9$ & 65,9 & 26066 & $+0,6$ \\
\hline 1993 & 3,2 & $-0,3$ & 2,9 & 2,9 & 0,0 & 67,0 & 25611 & $-1,8$ \\
\hline 1994 & 2,0 & 3,6 & 5,6 & 1,9 & $-3,7$ & 65,5 & 25242 & $-1,5$ \\
\hline 1995 & 2,2 & 2,2 & 4,4 & 3,1 & $-1,2$ & 64,5 & 25022 & $-0,9$ \\
\hline & & & & & & & & \\
\hline
\end{tabular}

Quellen: WSI (Hrsg.) Tarifpolitisches Taschenbuch, Ausgaben 1991 bis 1996, Köln; Jahresgutachten des Sachverständigenrates 1996, Bonn; eigene Berechnungen. 
Die verteilungs- oder beschäftigungsneutrale Lohnformel, wie sie von Meinhold 1965 aufgestellt und von Siebert 1994 aktualisiert wurde, ist allerdings eine interessengeleitete Konstruktion. Schöpft die Nominallohnerhöhung den so definierten Spielraum nicht aus, so verschieben sich die Verteilungsverhältnisse zugunsten der Kapitalseite. $\mathrm{Ob}$ sich daraus zusätzliche beschäftigungswirksame Investitionen ergeben, hängt von Faktoren ab, auf die die Tarifparteien keinen Einfluß haben. Wird der Spielraum überschritten, kann dies über die Verschiebung der Verteilungsverhältnisse zugunsten der abhängig Beschäftigten zu einer Steigerung des Konsums und darüber zu einem Anstieg der Investitionen führen. Auch die Steigerung der Produktivität ist nicht etwas Vorausgesetztes, das verteilt werden könnte, sondern das Resultat der Jahreswertschöpfung (BIP) pro Erwerbstätigen. Erst im nachhinein wird deutlich, ob die Verteilungsposition der Unternehmen ausgebaut oder geschwächt wurde. Es zeigt sich im Verlauf, daß den Gewerkschaften - ausgenommen 1970-72 - gesamtwirtschaftliche Verteilungserfolge regelmäßig am Ende des Konjunkturzyklus gelingen, wenn sie die gestiegene Nachfrage nach Arbeitskräften nutzen, um höhere Nominallöhne durchzusetzen, während sie die verteilungspolitischen Niederlagen in der Regel in den ersten beiden Jahren der Rezession hinnehmen müssen. In den 80er ist fast durchgängig eine negative verteilungspolitische Entwicklung zu verzeichnen, ein Teil des Lohnerhöhungsspielraums wird für die Arbeitszeitverkürzung geopfert. Diese bringt gesamtwirtschaftlich positive Beschäftigungseffekte und ist angesichts des Gesamtverlaufs plausibler als die Behauptung von der positiven Beschäftigungswirkung niedriger Lohnzuwächse. Ebenfalls sind die Beschäftigungsgewinne 1990/91 plausibler durch die »vulgärkeynesianische« Politik zunehmender Staatsverschuldung im Rahmen des Vereinigungsbooms zu erklären als durch niedrige Lohnsteigerungen.

Die Konstruktion eines kausalen Zusammenhangs von Lohnhöhe und Beschäftigungsgrad hält empirisch-orientierter und theoretischer Kritik nicht Stand (vgl, zuletzt Heise 1996) und muß daher als Versuch einer wissenschaftlich verbrämten Morallehre für ökonomisch korrektes Handeln kritisiert werden. Durch die Konfusion von mikroökonomischer Sichtweise mit makroökonomischen Zusammenhängen oder genauer: die Versimpelung komplizierter makroökonomischer Zusammenhänge auf einfache mikroökonomische Sichtweisen wirkt die Strategie durch niedrige Löhne zu mehr Beschäftigung zu kommen, plausibel. Was in den innergewerkschaftlichen Debatten über das »Bündnis für Arbeit « zur »Hegemonie über die Stammtische« verklärt wurde, war in Wirklichkeit die Reduktion gewerkschaftlicher Argumentation auf das Niveau des ökonomisch fachsimpelnden Stammtisches. Endlich wurde den Gewerkschaften die Anerkennung

5 Dessen Teilnehmer in erster Linie aus Wirtschaftsjournalisten und Politikern bestehen. 
der veröffentlichten Meinung zu Teil, die sie lange Jahre vermißt hatten, in der sie als Dinosaurier in sich rasch modernisierenden Industriegesellschaften abqualifiziert wurden.

Mit der überraschenden Übernahme der neoklassischen Lohn- und Beschäftigungstheorie war den Gewerkschaften in der Tat ein publizistischer Coup gelunden. Sie hatten ansatzweise aufzeigen können, daß großen Teilen der Unternehmensverbände die Ankündigung einer moderaten Tarifpolitik zur Sicherung der Flächentarifverträge nicht ausreichen würde und diese auf erheblich schmerzlicheren Anpassungsvorleistungen bestehen bleiben. In der politischen Öffentlichkeit wurde diese rigide Haltung gegenüber anpassungswilligen Gewerkschaften als nicht »fair« empfunden. Das politische System der Bundesrepublik ist noch so stark vom Geist der korporativen und kooperativen Beziehungen zwischen Kapital und Arbeit geprägt, daß die unvermittelte Aufkündigung des Kompromisses gegenüber einem bis zur politischen Selbstverleugnung bereiten Partner nicht ohne Einwände und Protest akzeptiert wird. Diese publizistische Unterstützung hat die Gewerkschaften bis zum Februar 1996 in ihrer Politik der Anpassung bestärkt. Erst mit der Umsetzung des 50-Punkte-Aktionsprogramms der Bundesregierung in konkrete Gesetzesinitiativen und der Verhandlungsblockade in den Tarifverhandlungen des Frühjahrs 1996, zunächst im öffentlichen Dienst, wurde den Gewerkschaftsführungen klar, daß ihre Kooperationsbereitschaft offen mißbraucht wurde. Dann erst begann der Umstieg auf Großdemonstrationen und Protest.

\section{Die Auseinandersetzung um die Öffnung der Flächentarifverträge}

In der Debatte über betriebliche Öffnungsklauseln für die Flächentarifverträge vermischen sich unterschiedliche Konzepte. Zum einen gibt es in allen Unternehmen mit Formen der Leistungsentlohnung ein Mitbestimmungsrecht in Fragen der betrieblichen Lohngestaltung. Zum zweiten wurde auch die Frage der konkreten Lage und der Verteilung der wöchentlichen Arbeitszeit auf die Arbeitstage betrieblich geregelt, da in den Tarifverträgen diese Fragen in der Regel nicht abschließend normiert waren und insofern Gestaltungsraum für betriebliche Entscheidungen unter Beteiligung der Betriebsräte bestand. Zusätzlich gab und gibt es in vielen Tarifverträgen weitere betriebliche Öffnungsklauseln. In den Bundestarifverträgen des Öffentlichen Dienstes kennen wir Öffnungsklauseln für regionale (bezirkliche und örtliche) und betriebliche Regeln. Das Gerede vom starren Korsett der Flächentarifverträge hat in den Tarifverträgen selbst keine sachliche Grundlage.

Unter der Leitidee einer über die Beteiligungsrechte des Betriebsrats hinausgehenden Beteiligung der Beschäftigten wurde in der Debatte über den 
mit dem Schlagwort Postfordismus umrissenen Umbruch in der industriellen Arbeitsorganisation versucht, zusätzlich zu den tariflichen Schutzbestimmungen betriebliche Vereinbarungen über die Gestaltung der Arbeitsbedingungen zu ermöglichen. Dabei wurde die betriebliche Beteiligung als Instrument der Anpassung von in der fordistischen Ära entstandenen Tarifverträgen an veränderte Bedingungen der Arbeitsorganisation verstanden (Riester 1994). In dieser Debatte ging es auch um die politische Bewertung dieses Umbruchsprozesses. Setzten die neuen Arbeitsformen und Arbeitsinhalte die Frage der Demokratie im Betrieb auf die Tagesordnung (MüllerJentsch 1994) oder handelte es sich um subtilere Strategien der Rationalisierung und der Subsumtion der Arbeit unter kapitalistischer Herrschaft? Wie in der Zunft der Arbeits- und Industriesoziologie gab es auch in den Gewerkschaften unterschiedliche Bewertungen, denen dann personalisierend die Etiketten Modernisierer (für die Gestaltungsoptimisten) und Traditionalisten (für die Skeptiker) angehängt wurden.

Spätestens mit dem Ende des 5. Nachkriegs-Konjunkturzyklus 1993/94 wurde diese Öffnungsdebatte durch die Einführung von Öffnungsklauseln überlagert, die durch weitergehende Arbeitszeitflexibilisierung und durch direkte Lohnsenkungen bzw. Arbeitszeitverkürzung ohne (vollen) Lohnausgleich auf eine betriebliche Arbeitskostensenkung zielten. Ungefähr zeitgleich verstärkte sich der Druck auf die Flächentarifverträge durch Verbandsflucht und gezielte Ausgründungen in Tarifbereiche mit niedrigerem Arbeitskostenniveau. Betriebliche Öffnungsklauseln oder Anwendungsvereinbarungen wurden einmal zugestanden, um die Wettbewerbsposition einzelner Unternehmen zu verbessern, zum anderen um Verbandsflucht oder Ausgründungen zu verhindern bzw. weder rückgängig zu machen. Damit wurde die Tarifpolitik der betroffenen Gewerkschaften direkt an die betriebliche Situation angebunden und in eine Strategie der betrieblichen Arbeitskostensenkung eingebunden. Zusammen mit der Idee des Bündnisses für Arbeit war dies ein doppelter Sieg der mikroökonomischen Sichtweise: Sie hatte sich gegenüber den Gewerkschaften sowohl ideologisch als auch praktisch durchgesetzt.

Für dieses Entgegenkommen in der Frage der Arbeitskosten gab es in der Regel Kompensationen: befristeten Kündigungsschutz, Teillohnausgleiche für niedrige Lohngruppen. Problematisch an dieser Öffnung der Flächentarifverträge ist jedoch einmal die Beschleunigung der Absenkung des Lohnniveaus, weil mit der Öffnung für betriebliche Anwendungen ein weitergehender Anpassungsdruck auf konkurrierende Unternehmen entsteht. Es wird somit eine Spirale nach unten in Gang gesetzt. Zum zweiten wird durch die Variante der Arbeitszeitverkürzung ohne Lohnausgleich schrittweise der bestehende Niedriglohnsektor ausgeweitet, da diese Arbeitszeit- 
verkürzung gezielt bei von Entlassungen bedrohten Beschäftigten durchgesetzt werden kann. Dies sind aber in größerem Umfang die geringer qualifizierten und deshalb niedriger entlohnten Arbeitskräfte. So gesehen ist diese Öffnung der Flächentarifverträge auch ein Instrument, um eine stärkere Lohnspreizung nach unten durchzusetzen. Dadurch würde die im internationalen Vergleich relative flache Lohnstruktur nach unten erweitert. Die in diesem Zusammenhang vertretene Behauptung, gerade die niedrigsten Löhne seien zu hoch und darum verantwortlich für die Arbeitslosigkeit (z.B. Streeck 1996) verwechselt Lohnhöhe mit Austauschbarkeit: Es werden die Arbeitskräfte entlassen, deren Tätigkeiten automatisiert werden können oder die durch noch billigere Arbeitskräfte ersetzt werden können. Es handelt sich um relativ gering qualifizierte Tätigkeiten, die in den Rahmentarifverträgen entsprechend niedrig bewertet werden. Geringe Qualifikation beinhaltet zugleich eine hohe Ersetzbarkeit und relativ niedrige Löhne. Eine weitere Spreizung der Löhne nach unten stärkt lediglich vorübergehend die Konkurrenzposition der Niedriglohngruppen gegenüber der Substitution durch Kapital und der Bedrohung durch die untertarifliche Konkurrenz. Die Spirale nach unten bleibt bestehen.

\section{Die Hoffnungen der angebotsorientierten Lohnsenkungsstrategie}

Hinter solchen und ähnlichen Vorstellungen - wie z. B. dem Vorschlag der Subventionierung niedriger Löhne durch eine negative Einkommensteuer steht die der Grenznutzentheorie immanente Grenzproduktivitätstheorie des Lohnes. Lohnkosten, die diese Grenzproduktivität niedrig qualifizierter Arbeitskräfte überschreiten, werden in dieser Sichtweise von den Arbeitskräften nachfragenden Unternehmen nicht mehr akzeptiert. Sollen solche Arbeitskräfte weiter nachgefragt werden, müssen die Löhne weiter abgesenkt und dann, um einen Lohnkostensenkungswettlauf zu vermeiden, subventioniert werden (Scharpf 1994). Die mikroökonomische Sichtweise von der Grenzproduktivität des Lohns verbindet Neoklassik und Standardkeynesianismus (einschließlich Keynes). Es kann daher auch, wenn makroökonomisch »keynesianisch«, also mit dem ernsthaften Ziel der Beschäftigungsausweitung argumentiert wird, mikroökonomisch für Lohnsenkung plädiert werden. Die Neoklassik will die Lohnsenkung, weil sie in ihrer Gleichsetzung von Güter- und Arbeitsmärkten einen »markträumenden « Lohn durchsetzen will, während ein »angebotspolitischer Keynesianismus « (Altvater 1988) auf eine Kombination von öffentlicher Beschäftigungsförderung durch einen staatlich alimentierten Niedriglohnsektor und gleichzeitige Marktimpulse durch abgesenkte Löhne setzt. Wird dazu noch mit der negativen Einkommenssteuer eine staatlich finanzierte Grundsicherung angeboten, entwickeln diese Konzepte trotz ihrer theoretischen Nähe 
zur neoklassischen Doktrin politische Attraktivität. Mit der finanziellen Entlastung der Sozialversicherung durch eine staatliche - also aus Steuermitteln finanzierte - Grundsicherung, können die Bruttoarbeitskosten (die »Lohnnebenkosten «) vermindert und es kann die Beitragsbelastung sowohl der Unternehmen als auch der abhängig Beschäftigten gesenkt werden. ${ }^{6}$ Davon versprechen sich die Gewerkschaften des exportorientierten Industriesektors eine zusätzliche Stärkung der internationalen Wettbewerbsposition ihrer Industrien und damit eine Sicherung ihrer Beschäftigungsperspektive. Relativ steigende Nettolöhne würden den insbesondere von der Bundesbank ausgeübten geldpolitischen Druck auf die Nominallohnentwicklung abschwächen und trotz geringer Nominallohnerhöhungen mindestens eine Reallohnsicherung ermöglichen. Diese Hoffnungen sind aber aus mindestens zwei Gründen trügerisch:

1. Angesichts des unterdurchschnittlichen Anstiegs der westdeutschen Lohnstïckkosten beinhaltet im internationalen Vergleich eine zusätzliche Stärkung (Ifo-Institut 1996, DIW 1995) der Wettbewerbsposition das Risiko einer weiteren DM-Aufwertung und bei politisch erzwungenen festen Wechselkursen kommt es zu einer Verstärkung der »beggar my neighbour« Politik gegenüber den wichtigsten Handelsländern.

2. Unter den gegebenen fiskalischen Bedingungen ist der Ausbau einer staatlichen Grundsicherung nur bei einer Verminderung der Leistungen der Sozialversicherung vorstellbar. Auch ein schrittweiser Umbau des Sozialstaats von der lohnbezogenen Umlagenfinanzierung wie sie das Sozialversicherungssystem kennzeichnet, auf eine steuerfinanzierte Umverteilung wird erhebliche Friktionen einschließen, da die Aufkommensleistung der direkten Steuern erheblich geringer als die des Sozialversicherungssystems sein wird und eine kräftige Anhebung indirekter Steuern nicht nur verteilungspolitisch unsozial wirkt, sondern auch die Inflation fördern würde, was die Bundesbank zur Verschärfung ihrer restriktiven, ausschließlich auf Geldwertstabilität fixierten Geldpolitik motivieren dürfte.

Mit der Revision der frühen grundsätzlichen Position des »Lohnverzicht schafft keine Arbeitsplätze« konnte sich die angebotspolitische Orientierung in der Tarifpolitik in den Gewerkschaften weitgehend durchsetzen.

6 Darauf zielt die Debatte um die Steuerfinanzierung sog. versicherungsfremder Leistungen der Sozialversicherung. Angesichts eines im internationalen Vergleich unterdurchschnittlichen Anstiegs der westdeutschen Lohnstückkosten, in denen die gesamten Bruttoarbeitskosten enthalten sind (siehe DIW 1995, Ifo-Institut 1996), ist es ökonomisch absurd, einen Teil dieser Lohnstückkosten, als sog. Lohnnebenkosten für zu hoch zu erklären, wie dies jüngst wieder der DGB-Vorsitzende Schulte getan hat. Ebenso absurd ist es, sich von einer Senkung der Sozialbeiträge, eine Steigerung der konsumtiven Nachfrage zu versprechen, da im deutschen System der Umlage- - und eben nicht Kapitalstockfinanzierung - die Sozialbeiträge direkt in die konsumtive Nachfrage und zwar als Renten, Arbeitslosenunterstützung, Krankengeld eingehen. 
Schon zu Beginn der 80er hatte eine damals noch offen kapitalismuskritisch sich artikulierende Sozialwissenschaft dies als Anpassungsleistung der deutschen Gewerkschaften an eine verschärfte Weltmarktkonkurrenz kritisiert (Esser 1992, 224). Die These von der neokorporatistischen Blockbildung zur Modernisierung der (westdeutschen) Volkswirtschaft greift heute, da sich große Teile des Unternehmerlagers der Blockbildung verweigern, offenkundig zu kurz. Aber schon damals hatte sie den beteiligten Gewerkschaften strategisches Verhalten zur Sicherung des Wirtschaftsstandorts unterstellt, das vielleicht für Minderheiten in den Gewerkschaftsapparten angemessen war, aber die Tarifpolitik der Gewerkschaften nicht hinreichend erklären konnte. Anfang 1988 hatte die inhaltlich auch »neoklassisch « argumentierende Initiative Lafontaines in Richtung Arbeitszeitverkürzung ohne Lohnausgleich die harsche Kritik gerade der Gewerkschaftsintellektuellen ausgelöst, die heute die Bündnis-Offerte argumentativ vor- und nachbereitet haben.

Die Bündnis-Initiative zielt auch arbeitspolitisch nicht mehr auf die Sicherung und gegebenenfalls den weiteren Ausbau der Exportposition der deutschen Unternehmen. In ihr geht es im Kern darum, bei veränderten politisch-ökonomischen Rahmenbedingungen, die sowohl die Organisation der Lohnarbeit, als auch die makroökonomische Ebene betreffen, ein »bewährtes « Modell der Arbeitsbeziehungen auf betrieblicher wie überbetrieblicher Ebene fortzusetzen, m. a. W. die deutschen Gewerkschaften in ihrer bisherigen Funktion als Partner in einem Konsensmodell zu erhalten bzw. die politischen Rahmenbedingungen für die Fortsetzung der Konsenspolitik neu auszutarieren. Dies erfordert die Öffnung und Flexibilisierung tariflicher Regeln und die Preisgabe der traditionellen tarif- und sozialpolitischen Grundsätze. Dieser ideologische Kurswechsel in einem politischen Anpassungsprozeß hat ganz praktische Vorteile: mit ihm können die pragmatische Tarifarbeit, also das »Abspecken« der eigenen Tarifverträge, ebenso wie die politisch erzwungene Preisgabe der früheren Handlungsspielräume in der Sozialversicherung legitimiert werden. Die grundsätzliche Entscheidung für die Fortsetzung der Konsensstrategie auch um den Preis weiterer verteilungspolitischer Niederlagen war schon in den Diskussionen zum neuen Grundsatzprogramm des DGB deutlich geworden, als in den ersten programmatischen Positionspapieren - sog. Leitbildern - die Vision einer auf Kommunikation, Dialog und letztlich Konsens angelegten "Zivilgesellschaft « skizziert wurde. Trotz der mit Sicherheit eintretenden Zunahme der (saisonbereinigten) Arbeitslosigkeit, trotz der nachweisbaren Erfolglosigkeit einer Tarifpolitik der Lohnzurückhaltung - die bereits in den 80er Jahren vorgeführt wurde - werden die Gewerkschaften in der absehbaren Zeit diese Politik fortsetzen. Der vorhandenen Chance, in der Auseinanderset- 
zung um die Entgeltfortzahlung durch einen Arbeitskampf zugleich einen tarifpolitischen und einen gesellschaftspolitischen Erfolg zu erzielen, wurde mit großer Energie in mehreren Anläufen ausgewichen.

Auf eine Phase erheblich härterer betrieblicher und gesellschaftlicher Konflikte fühlen sich die Gewerkschaftsführungen und die große Mehrheit der aktiven Mitglieder mental und organisatorisch nicht ausreichend vorbereitet. Das hängt auch damit zusammen, daß die Wahrnehmung der Entwicklungschancen des deutschen Kapitalismus und der darin angelegten Handlungsmöglichkeiten der Gewerkschaften zwischen 1988 und 1992 überwiegend optimistisch gewesen ist. Sowohl der postfordistische Umbruch in der Arbeitsorganisation als auch der rasche Niedergang der staatssozialistischen Gesellschaftssysteme wurden als Belege einer »zivilgesellschaftlichen « Entwicklungsdynamik zumindest des deutschen Kapitalismus interpretiert und die Beteiligungschancen der abhängig Beschäftigten in diesem Veränderungsprozessen wurden erheblich überschätzt ${ }^{7}$. Mit der Rezession 1993/94 wurden diese Hoffnungen faktisch desillusioniert. Die Bündnis-Offerte erfolgte daher aus der Position einer tiefgehenden Verunsicherung. Die Bündelung der gegenwärtigen Umbruchsprozesse - post- bzw. neotayloristischer Formen der Arbeitsorganisation, eine mittelfristige Stagnation der Realkapitalakkumulation bei gleichzeitiger Dominanz der Finanzmärkte und eine restriktive, beschäftigungsfeindliche Geld- und Fiskalpolitik von Zentralbank und Staat, verstärkt durch den aktuellen Angriff auf Essentials des Arbeits- und Tarifrechts - erklärt, warum sich die Gewerkschaften nach der vorläufigen Abwehr dieser Angriffe nicht auf ein Überdenken ihrer gescheiterten Strategie, sondern für die Fortsetzung entscheiden. In der Konsequenz beinhaltet dies die Preisgabe des traditionellen Anspruchs der Gewerkschaften, einen politischen Zusammenhang zwischen Beschäftigten und Arbeitslosen herzustellen und gesamtwirtschaftlich für eine Vollbeschäftigungsstrategie einzutreten. Nationale Lohnzurückhaltung verbessert lediglich die internationale Wettbewerbsposition und verschärft darüber den deflationären Wettlauf zwischen den Industrieländern der Triade einerseits und den europäischen Ländern andererseits. In der Einbindung in diese Globalisierungsfalle zeigt sich der Konservatismus deutscher Gewerkschaften. Sie sind gegenwärtig nicht in der Lage, auf die Strukturveränderungen innerhalb des modernen Kapitalismus mit einer Än-

7 Beteiligung ist ein zentrales Schlüsselwort der modernen Gewerkschaftssoziologie - damit verbunden ist die Hoffnung der Umbruch in der Arbeitsorganisation würde auch das überlieferte Herrschaftsverhältnis von Kapital und Arbeit in Betrieb und Gesellschaft in Richtung einer stärkeren Partizipation der abhängig Beschäftigten modifizieren. Die davon berührte tradierte Form der betrieblichen und gewerkschaftlichen Interessenvertretung wird zugunsten einer stärker individuell orientierten Beteiligung kritisch in Frage gestellt und die Schutzfunktion kollektiver Interessenvertretung relativiert. 
derung ihres strategischen Verhaltens zu reagieren, sondern versuchen, mit ihrer traditionellen Vorstellung einer »intermediären « Interessenvertretung (Müller-Jentsch 1982) auch in den 90er Jahren über die Runden zu kommen. Das bereits absehbare Scheitern dieses Versuchs wird die internen Gegensätze in den Gewerkschaften und zwischen einzelnen Gewerkschaften offen auftreten lassen.

\section{Literatur}

Altvater, Elmar (1988): Nationale Wirtschaftspolitik unter Bedingungen globaler finanzieller Instabilitäten - zu Fritz Scharpf's »angebotspolitischen Keynesianismus« in: PROKLA 72.

Altvater Elmar; Mahnkopf, Birgit (1993): Gewerkschaflen vor der europäischen Herausforderung, Münster.

Deutsches Institut für Wirtschaftsforschung (1995): Hat Westdeutschland ein Standortproblem? in: DIW-Wochenbericht 38/96.

Heise, Arne (1996): Arbeit für Alle - Vision oder Illusion, Marburg.

Ifo-Institut (1996): Sind Löhne und Steuern zu hoch? In: Ifo-Schnelldienst 20/96.

Keynes, John Maynard (1973): Allgemeine Theorie der Beschäftigung, des Zinses und des Geldes, München.

Lang, Klaus; Kuhlmann, Reinhard (1996): Bündnis für Arbeit - Kristallisationspunkt und Reformperspektive für Vollbeschäftigung, in: Gewerkschaftliche Monatshefte 3'96.

Marx, Karl (1867): Das Kapital Bd. 1, in: MEW Bd. 23.

Müller-Jentsch, Walter (1994): Industrielle Demokratie - von der repräsentativen Mitbestimmung zur direkten partizipation, in: Gewerkschaftliche Monatshefte 6'94.

- (1982): Gewerkschaften als intermediäre Organisationen, in: Brandt, Gerhard; Jacobi, Otto; Müller-Jentsch, Walter, Anpassung an die Krise: Gewerkschaften in den siebziger Jahren, Frankfurt/M.

Riester, Walter (1994): Tarifpolitik im Umbruch - Tarifpolitik und Beteiligung, in: Oetjen, Hinrich; Zoll, Rainer (Hrsg.) (1994), Gewerkschaften und Beteiligung, Münster

Scharpf, Fritz W. (1993): Von der Finanzierung der Arbeitslosigkeit zur Subventionierung niedriger Erwerbseinkommen, in: Gewerkschaftliche Monatshefte 7'93.

Esser, Josef (1982): Gewerkschaften in der Krise, Frankfurt/M. 1982.

Glastetter, Werner; Högemann, Günter; Marquardt, Ralf (1991): Die wirtschaftliche Entwicklung in der Bundesrepublik Deutschland 1950 - 1989, Frankfurt/M.

Siebert, Horst (1994): Geht den Deutschen die Arbeit aus? München.

Streeck, Wolfgang (1996): Anmerkungen zum Flächentarifvertrag und seiner Krise, in: Gewerkschaftiche Monatshefte 2'96.

Wendl, Michael (1996a): Aktionsprogram für weniger Arbeit, in: Sozialismus extra 1996.

- (1996b): Öffnung nach unten - Risiken einer weiteren Verbetrieblichng der Tarifpolitik, in: Sozialismus 12/96. 